\title{
Saltando la brecha: el desarrollo de un producto innovador desde la perspectiva del análisis de redes sociales
}

\author{
Jumping the Gap: developing an innovative product \\ from a Social Network Analysis perspective
}

\section{Leandro Martín Ferron}

CONICET, Argentina. Email: leaferron@hotmail.com

\section{RESUMEN}

La creación de nuevos productos a partir de la vinculación entre ciencia e industria posee aspectos que pueden ser revisados atendiendo a la naturaleza de los vínculos academia-empresa, a la estructura de los sistemas de Ciencia y Tecnología o a los modelos de articulación academia-industria. Esta investigación propone un abordaje novedoso que toma como base el análisis de un caso de estudio y utiliza la perspectiva del análisis de redes sociales para reconstruir los caminos académicos-estatales-empresariales necesarios para la generación de una innovación. La fuente principal de datos fue el relato de un informante clave, tomando al producto innovador como ego de la red. Se identificaron las vías de circulación de conocimiento, así como subgrupos de nodos cuyos atributos están asociados a las acciones de transferencia, desarrollo y financiamiento del producto. El actor con mayor grado de centralidad de la red pertenece al sector académico y opera como puente que vincula los sectores académico y estatal, lo que podría ser indicio de que la promoción de este tipo de perfiles dentro del sector académico podría traccionar el desarrollo de productos innovadores con base en conocimiento científico.

Palabras Clave: vinculación academia-industria, transferencia tecnológica, producto innovador, análisis de redes sociales.

\begin{abstract}
The creation of new products from the bonding between science and industry incorporates aspects that can be reviewed based on the nature of the academy-business bonds, the structure of the science \& technology systems, or the models of academy-industry articulation. This research proposes a novel approach based on the analysis of a case study and uses the perspective of social network analysis to reconstruct the academic-state-business links necessary for the generation of innovation. The main source of data was the narration of a key informant, taking the innovative product as the ego of the network. Knowledge circulation pathways were identified, as well as subgroups of nodes whose attributes are associated with the product's transfer, development, and financing actions. The actor with the highest degree of centrality in the network belongs to the academic sector, and he operated as a bridge that bonded the academic and state sectors. This could be an indication that the promotion of this type of profile within the academic sector could boost the development of innovative products based on scientific knowledge. Keywords: academy-industry bonding, technology transfer, product innovation, social network analysis.
\end{abstract}

Como citar: Martín Ferron, L. (2021). Saltando la brecha: el desarrollo de un producto innovador desde la perspectiva del análisis de redes sociales. AWARI, 2, e026. https://doi.org/10.47909/awari.128

Recibido: 12-03-2021 / Aceptado: 14-07-2021

Editor: Alejandro Paredes

Copyright: $\odot 2021$ The author(s). This is an open access article distributed under the terms of the CC BY-NC 4.0 license which permits copying and redistributing the material in any medium or format, adapting, transforming, and building upon the material as long as the license terms are followed. 


\section{INTRODUCCIÓN}

S EGÚN Rosenberg (1976), la generación de innovaciones constituye un proceso complejo, determinante en el crecimiento y desarrollo de las economías, y cuya intensidad depende en gran medida de la capacidad de absorción y adaptación de las unidades empresariales. En la Argentina, a pesar del creciente interés que muestran los decisores de políticas públicas en la promoción de estrategias para la generación de innovaciones, existen algunas áreas en las que el conocimiento sobre la problemática de la creación de nuevos productos surgidos a partir de la vinculación entre ciencia e industria es aún limitado. Algunos de estos aspectos pueden ser revisados atendiendo a la naturaleza de los vínculos academia-empresa, a la estructura de los sistemas de Ciencia y Tecnología (CyT) o a los modelos de articulación academia-industria. Si bien es cierto que el estudio de las interacciones academia-industria ha sido objeto de vastas investigaciones (Mansfield, 1991; Laursen y Salter, 2004; Brimble y Florida, 2007; Segarra-Blasco y Arauzo-Carod, 2008; Tether y Tajar, 2008), existen aún hoy visiones contrapuestas sobre cuál debe ser el papel del sector científico y cuál es el modelo de relación academia-industria que presenta mejores resultados en lo concreto.

Por una parte, el modelo de Triple Hélice postula estrechar la integración entre empresas y academia, hasta el punto de que esta última incluso adopte prácticas empresariales a fin de maximizar la capitalización del conocimiento, deviniendo en un modelo de Universidad-Empresa cuya meta es la comercialización del conocimiento generado en la academia a través de patentes, licencias y la creación de spin off (Etzkowitz et. al., 2000). En contraste, los académicos que sostienen la tercera misión de la universidad como una forma de ver y entender la investigación e innovación, impulsar el desarrollo y promover la transmisión fuera del ámbito universitario, proponen una colaboración conjunta entre empresas, Estado, universidad y sociedad (Galindo et al., 2011), que se puede reflejar en los servicios de investigación $\mathrm{y}$ asesoramiento a organismos gubernamentales y no gubernamentales (Molas-Gallart, 2005), y cuyas actividades van más allá de la transferencia de conocimiento, debiendo incluir el desarrollo de iniciativas de aprendizaje permanente y servicio público (de la Torre et. al., 2017). Este abordaje se opone al modelo de la "Universidad-Empresa" mediante el argumento de que este esquema desdibuja el rol de contribución al cambio y al desarrollo que las universidades deben sostener (Arocena y Sutz, 2005; Gulbranddsen y Slipersaeter; 2007).

En el contexto latinoamericano, se observa que la mayoría de las políticas y acciones implementadas en torno a la generación de innovaciones han estado basadas en la experiencia de sistemas científicos con mayores recursos, tomando como referencia las capacidades que los sectores generadores de conocimiento tienen en esos países y abordando con escasa frecuencia el análisis de los vínculos academia-empresa en regiones con economías en desarrollo o desde perspectivas alternativas como pueden ser la de las empresas, la de los investigadores, la de organismos del estado o la de las comunidades involucradas en las acciones de vinculación (Cohen et. al., 2002).

En este marco, nuestro trabajo propone un abordaje novedoso que consiste en llevar a cabo una "ingeniería inversa" del entramado de relaciones requeridas para el surgimiento de un producto innovador. Así, tomando como base un caso de estudio, buscamos reconstruir desde la perspectiva del análisis de redes sociales (ARS) los caminos académicos-estatales-empresariales que fueron necesarios recorrer para la generación de una innovación a partir de acciones de transferencia de conocimientos desde la academia al sector productivo. El objetivo es identificar qué actores debieron ser partícipes, qué roles ocuparon y de qué forma debieron relacionarse para concretar el desarrollo de una innovación, intentando identificar cuál hubiera sido el trayecto más corto para que el conocimiento académico devenga en un producto de valor para las empresas.

\section{CONTEXTO DE ANÁLISIS}

Se tomó como caso de estudio un sistema innovativo para iluminación natural destinado al mercado de la construcción en la Argentina. El uso de este dispositivo está orientado a viviendas residenciales, y fue desarrollado a partir de una experiencia de vinculación academia-empresa. El grupo de investigación desde 
el qué se realizó la transferencia de conocimiento es el Instituto de Ambiente, Hábitat y Energía (INAHE), inserto en el Centro Científico Tecnológico Mendoza (CCT Mendoza), perteneciente al Consejo Nacional de Investigaciones Científicas y Técnicas (CONICET), Argentina.

El investigador responsable de la transferencia (LF) se vinculó con el CONICET a través del Laboratorio de Ambiente Humano y Vivienda (LAHV) del Instituto de Ciencias Humanas, Sociales y Ambientales (INCIHUSA) el año 1997, cuando todavía era estudiante de grado de la carrera de Diseño Industrial en la Universidad Nacional de Cuyo (UNC), gracias a la invitación que un docente de 3er año hizo a una de las investigadoras del instituto (AP) como asesora para la realización de un práctico de cátedra, relacionado con el diseño de ventanas energéticamente eficientes.

Esta interacción propició que un grupo de estudiantes (en el que estaba LF) se acercara espontáneamente al entonces denominado CRICYT-CONICET (antiguo nombre del CCT Mendoza) para ofrecerse como colaboradores voluntarios en proyectos de diferentes líneas. Ese mismo año, se les encomendó resolver, bajo la dirección de la investigadora invitada a la cátedra (AP), el diseño de un lumiducto vertical para ser instalado en una escuela rural en la que el grupo de trabajo del LAHV estaba interviniendo como proyectista. En noviembre de 1998, el grupo de pasantes participa con una publicación sobre el diseño del lumiducto en el encuentro anual de la Asociación Argentina de Energía Solar (ASADES).

En el año 2005, LF postula y gana una beca doctoral CONICET presentándose con AP como directora, en el tema "Diseño y desarrollo de sistemas innovativos de iluminación natural". El año siguiente, también gana una beca doctoral ME, otro diseñador industrial, egresado de la UNC, 7 años menor que LF. Ambos cursan los seminarios de doctorado en la Universidad Nacional de Tucumán (UNTucuman), hasta el año 2007. En ese momento, ME abandona la beca para trabajar en una fábrica de cerámica recuperada llamada COLBO. En el año 2010 LF presenta su tesis doctoral, habiéndole dado especificidad a su tema en torno al desarrollo y caracterización de "Barras de iluminación natural".
Durante el desarrollo de su tesis doctoral y de su beca posdoctoral, LF trabajó en el diseño de sistemas de lumiductos verticales, indagando tecnologías y capacidades disponibles de la industrial a nivel local, vinculándose con diferentes talleres metalúrgicos y de trabajo en acrílico para la fabricación de modelos y el establecimiento de vínculos académico-empresariales. Adicionalmente, realizó múltiples modelos e instalaciones de sistemas de lumiductos verticales en residencias particulares, con el fin de probar estos sistemas en situación de uso real.

En 2013 LF ingresa a la carrera de investigador de CONICET, y con la experiencia adquirida en su etapa como becario tiene la intención de llevar del desarrollo de un sistema de lumiductos al mercado, mediante la vinculación con alguna empresa de la provincia que aborde la fabricación seriada de los dispositivos bajo licencia de CONICET. Sin embargo, la necesidad de presupuesto y de recursos humanos requeridos para adaptar los modelos de laboratorio a la escala industrial resultan una barrera que no permite establecer vínculos con empresas que se comprometan a invertir en I+D del proyecto.

En el mes de octubre de 2015, el LAHV se constituye en una nueva Unidad Ejecutora, el Instituto de Ambiente, Hábitat y Energía (INAHE); creada por Resolución D. CONICET $\mathrm{N}^{\circ} 4136 / 15$, cuya dirección es ganada por concurso por AP.

Ese año, LF realiza una estancia de investigación en el Instituto de Arquitectura Avanzada de Cataluña (IAAC, España), donde propuso el desarrollo de sistemas innovativos de iluminación, pero al iniciar el trabajo acuerda con los directivos académicos de la institución abordar el desarrollo de un nuevo tipo fuente de iluminación blanca basada en LASER. Un vez finalizada la estancia, y fundado en los lazos académicos generados con el IAAC, el investigador presentó en 2016, en dos oportunidades, un proyecto para ser financiado de manera bilateral comprometiendo la participación de cinco instituciones: la empresa Santa\&Cole , el IAAC2 y al Centro de Desarrollo de Sensores, Instrumentación y Sistemas, por España3, y al CCT-Mendoza4 y a la empresa MACAR5 (especializada en desarrollos metalúrgicos para la industria petrolera) por Argentina. En ambas 
ocasiones la postulación obtuvo dictamen favorable por parte del organismo de financiamiento de España (CYTED), y dictamen negativo por parte de los evaluadores de Argentina (MINCYT), perdiendo la posibilidad de continuar esa línea de desarrollo.

De vuelta en Argentina, en septiembre de 2016 LF participa en calidad de asistente de la presentación de líneas de financiamiento ofrecidas por el Consejo federal de Ciencia y Tecnología (COFECYT) para proyectos de aplicación de tecnologías. Interesado en la temática, se pone en contacto con la principal expositora, la directora del organismo, RO, consultando por una línea de financiamiento orientada a los gobiernos provinciales para la contratación de recursos humanos para la formulación y gestión de proyectos COFECYT (línea Vinculadores Tecnológicos Federales - VITEF). Por recomendación de $\mathrm{RO}$, el investigador se contacta con el director de desarrollo e innovación productiva de la provincia, LN, para postularse como VITEF para Mendoza. La postulación es aprobada y LF comienza a cumplir el doble rol de investigador de CONICET y vinculador tecnológico del COFECYT.

En diciembre de 2016 LF contacta a la empresa ENERGÉ, referente en la fabricación y venta de termotanques solares en Mendoza, surgida a partir de un proceso de incubación de la UNCuyo. Propone la fabricación de lumiductos, pero la propuesta es rechazada. Se propone entonces la solicitud de un financiamiento conjunto al COFECYT para el desarrollo de lumiductos bajo licencia CONICET-ENERGÉ. La propuesta vuelve a ser rechazada, pero la empresa propone la fabricación e instalación de cuatro lumiductos de gran tamaño en su nuevo showroom. LF acepta proyectarlos, fabricarlos e instalarlos, con el objetivo de generar entusiasmo con el producto. Luego de finalizada la obra, la empresa siguió sin aceptar abordar un desarrollo conjunto.

En marzo de 2017 el INAHE decide hacer una presentación formal en sociedad con la intención de dar a conocer las líneas de investigación y las capacidades del instituto a instituciones, empresas, referentes de la esfera política y al medio socio-productivo en general. Entre los invitados a la reunión (con formato exposición/ ágape), participan los socios de ENERGÉ y socios de la empresa Soluciones Constructivas, una empresa cuyo núcleo de negocio se basa en la prestación de servicios de perforación a la industria petrolera que estaba incursionando en servicios para la construcción. En esa instancia LF conoce a DG, socio-gerente de la empresa, que manifiesta que la empresa participó del evento porque estaba en la búsqueda de tecnologías "verdes" para incorporar como ventaja competitiva a su nueva línea de negocio.

En agosto de ese año, se abre nuevamente la ventanilla de financiamiento de los proyectos COFECYT. LF organiza jornadas de difusión y capacitación sobre las líneas disponibles, convocando a diferentes sectores académicos y estatales. Asisten a esa reunión los responsables de gestión ambiental del departamento de Alvear, Mendoza, que se contactan con LF con la inquietud de instalar dispositivos de iluminación en una planta de recuperación de plásticos del departamento.

LF formula la idea proyecto para la solicitud de financiamiento al COFECYT, pero el municipio de Alvear manifiesta no disponer de fondos para aportar la contraparte (30\% dinerario). Con la intención de avanzar con la presentación, LF propone a la empresa Soluciones Constructivas solicitar el subsidio, contra presentación de la contraparte, con el objetivo de dotar al municipio de sistemas de iluminación natural y con el beneficio de participar en el desarrollo de estos dispositivos de manera conjunta con el CONICET. La empresa acepta la propuesta.

El proyecto fue presentado en septiembre de 2017, aprobado en marzo de 2018 y recibió el primer desembolso ( $40 \%$ del valor total solicitado) en septiembre de 2019. El plazo transcurrido desde la cotización y solicitud del proyecto hasta el desembolso efectivo del primer adelanto representó en una devaluación de la moneda de un $80 \%$, por lo que fue necesario cambiar el plan de trabajo previsto, en el que se proponía la compra de maquinaria para la mecanización láminas de metal y materiales acrílicos, por una estrategia de desarrollo de piezas que pudieran ser fabricadas por terceros. También se debió plantear un cambio de tecnología, pasando de la chapa estampada a un material más económico, barajando la posibilidad de utilizar tecnologías de bajo costo como materiales cerámicos, factibles de ser producidos por moldeo o por colada. 
LF decide consultar a ME, que en ese momento ya es socio y responsable de la fábrica de Cerámica COLBO, sobre los alcances y limitaciones de esa tecnología para la fabricación de piezas de gran tamaño. ME sugiere una solución menos compleja debido a la poca estabilidad dimensional y el requerimiento de cocido de las piezas, planteando el uso de tecnologías de hormigón. LF estableció entonces contacto con la empresa CIMALCO, fabricantes de mobiliario de hormigón, radicada en Mendoza, pero la situación de contexto de pandemia hizo que el diálogo se extendiera en el tiempo y que finamente el proyecto no resultara de interés para la compañía. Frente a esta situación, LF decidió buscar otro posible proveedor a nivel nacional, llegando así a la empresa DURBAN en marzo de 2020, radicada en Santa Fe, que manifestó su interés en el proyecto, y fabricó en agosto 2020 el primer molde y prototipo de la pieza de colección de luz del dispositivo.

\section{MÉTODO}

Esta investigación sigue la metodología del caso de estudio (Yin, 2014), elegida en primer lugar debido al carácter único que suelen poseer las innovaciones tecnológicas, al menos en sus primeras fases $y$, en segundo lugar, por el hecho de que el concepto de innovación es relativo al entorno en el que se produce como fenómeno. Tomando como referencia inicial el ámbito de las energías renovables en el contexto de un país latinoamericano, se implementó un abordaje de análisis de redes sociales en torno a un producto que puede ser considerado tecnológicamente innovador, un dispositivo para la iluminación natural cenital de espacios residenciales.

\subsection{Recolección de datos}

El caso de estudio seleccionado se eligió por tratarse un producto tecnológico innovador, surgido de conocimientos desarrollados en la academia y basado en el uso de tecnologías endógenas. La fuente principal de datos fue el relato de LF (considerado informante clave) sobre los actores, las relaciones y las acciones llevadas a cabo en torno a la gestación del producto, desde la etapa de formación de los RRHH y de generación de conocimiento hasta el desarrollo del prototipo y su fabricación en serie. Esto comprende tanto preguntas acerca del producto (su clasificación, fuentes de financiamiento, conformación, mercado, tecnologías y antecedentes) como preguntas hacia los alter (tipo de nodo, tipo de aporte, tipo de participación, beneficios que obtiene, compromisos que asume). De la información recolectada se utilizaron los datos relativos a los egos y a la red de relaciones percibida entre actores, ya sean individuos, instituciones o empresas (enfoque egocéntrico).

\subsection{Análisis de la información}

El análisis cualitativo del trabajo se aborda desde un enfoque egocéntrico y gira en torno a las preguntas respecto del tipo de relación que cada alter tiene con el desarrollo tecnológico, tomado éste como Ego a partir del cual se construye la red. En el ARS los estudios atributivos no suelen ser la parte central de una investigación, puesto que el factor explicativo principal son las pautas relacionales (Lozares, 1996), pero, cuando se analizan las primeras fases de la constitución y puesta en marcha de sistemas relacionales, este tipo de estudio ayuda a sentar las bases de la tipología de estructura de la red social para llevar a cabo, posteriormente, el análisis relacional.

La recolección de información y la conformación del modelo de análisis estuvieron orientados por las preguntas de investigación planteadas y en la conformación de las redes propuestas. Al respecto, el proceso de investigación tuvo tres pasos lógicos: 1) una instancia de conformación de la red, estructurada a partir de información cualitativa, 2) una segunda instancia de análisis de las métricas, arrojando resultados netamente cuantitativos y, finalmente, 3) una tercera instancia de reflexión y profundización del análisis teniendo en cuenta los resultados de los pasos anteriores.

1) El tipo de nodo y el tipo de lazo de interés estuvieron definidos desde el principio, permitiendo identificar los límites y la estructura de la red desde un inicio. En este sentido, los nodos que componen la red se corresponden con personas, instituciones o empresas y cada lazo señala el vínculo de transmisión de conocimiento, recursos o acciones en torno a la generación de la innovación.

2) Para avanzar sobre el segundo paso en la investigación, se seleccionaron las métricas más relevantes que permiten reflexionar sobre 
la transferencia de conocimiento en términos estructurales. Así, se obtuvieron las medidas de centralidad de los nodos (de grado, de intermediación y de cercanía). Además, se consideraron las medidas de red completa como la densidad, el tamaño y los subgrupos conformados a partir del algoritmo de Girvan -Newman.

3) El proceso del análisis que tuvo lugar en este tercer paso apoya la afirmación de Teves cuando dice que "el ARS organiza la información de manera que la contextualización de la conducta de un actor y, en particular de las interacciones de un grupo de actores, pueden observarse y entenderse en base a las posibilidades y restricciones de una estructura la cual opera a múltiples niveles" (2011:25). Es en la configuración de estos múltiples niveles donde reside la fuerza de la noción de estructura, es decir que la configuración topográfica de las redes permite ubicar estructuralmente a los nodos más allá de sus características individuales $y$, de esta forma, agrega información a la ya relevada e integra las características individuales para dar un salto en la escala del análisis hacia un nivel general (Mardsen 1990).

\section{RESULTADOS}

La pregunta planteada para el análisis y construcción del grafo indaga sobre la existencia de relaciones entre los actores, previas al desarrollo de la innovación (sin especificar su naturaleza), identificando como atributos para cada nodo el sector al que pertenece, si contribuyó con financiamiento, know-how, acciones de gestión, si obtendría algún rédito, y cuál fue su aporte más relevante (incluyendo RRHH, fabricación, conocimientos, dinero y poder de gestión). La red resultante está compuesta por 33 actores, entre los que encontramos:

- 13 personas (académicos, empresarios, funcionarios y profesionales),

- 5 empresas,

- 3 instituciones educativas,

- 5 organismos nacionales,

- 1 organismo provincial y

- 6 programas de financiamientos

Los sectores a los que pertenece cada actor se representan en el Gráfico 1 diferenciados por color: rojo para el sector académico, azul el sector privado y negro los organismos estatales. La distribución de los nodos responde al ordenamiento de grupos por atributos, destacando en este caso el atributo Aporte más relevante, el tamaño de los nodos está determinado por la métrica Grado de centralidad, y la forma asignada a cada uno se corresponde con el atributo Aporte más relevante.

Las medidas estructurales de la red arrojan un diámetro igual a 3 y una densidad de grafo de 0.262 . Esto significa que el promedio de pasos necesarios para ir de un nodo a otro es de 3 , al tiempo que se observa una densidad entre baja, lo que indica que se trata de una red poco conectada.

Por otro lado, la red presenta dos subgrupos fácilmente identificables, ambos compuestos por nodos pertenecientes a los tres sectores - académico, privado y estatal- pero diferenciados por su ubicación relativa en el grafo. El primero de los sub-grupos se posiciona en la esquina superior derecha, mostrando un conjunto de actores con un mayor grado de centralidad, lo que confirma que tienen relaciones previas al desarrollo del producto innovador. Asimismo, dentro de este subgrupo los actores con más presencia y con mayor peso en términos de grado, esto es, el número de nodos a los que un actor está directamente unido, pertenecen al sector académico. Sin embargo, en cuanto al tipo de contribución directamente implicada en el desarrollo de la innovación (indicada por la forma) observamos que los nodos involucrados no realizaron aportes significativos. En segundo lugar, encontramos un subgrupo de nodos distribuidos en forma de medialuna sobre el margen inferior del grafo. Este subgrupo muestra un grado menor de centralidad, y a pesar de que también está compuesto por actores pertenecientes a los tres sectores, en oposición al primer grupo el sector académico está minoritariamente representado por un único nodo. A pesar de esto, este único nodo académico destaca por poseer el mayor grado de centralidad, tanto del subgrupo como de toda la red. Con respecto la contribución de cada nodo podemos identificar aportes diversificados que comprenden financiamiento, fabricación, know-how, gestión y RRHH. En términos concretos, este subgrupo comprende a los actores cuya participación e interacción fue estrictamente necesaria para el desarrollo del producto innovativo. 


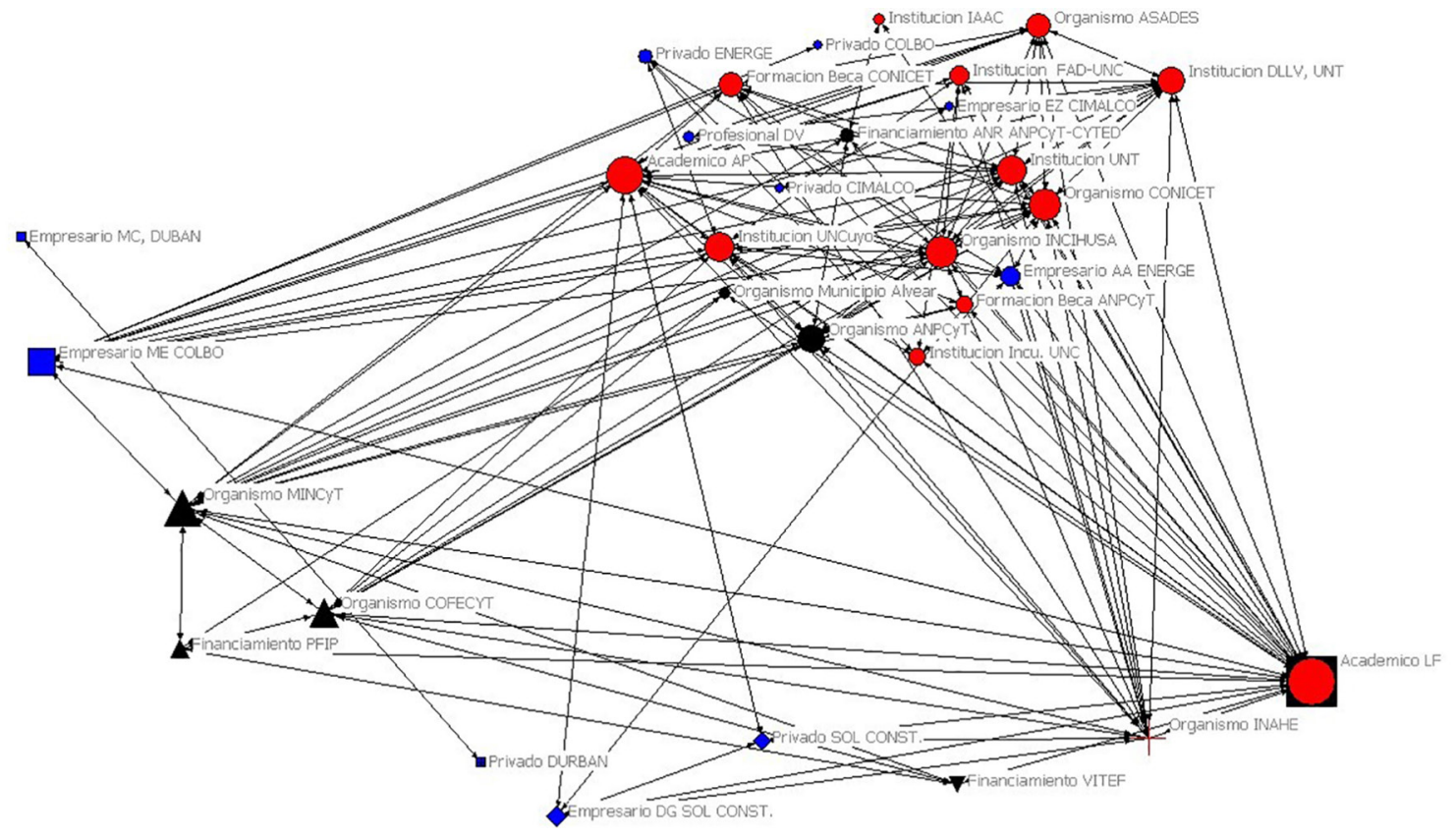

Gráfico 1. Red completa de alteri.

Centrándonos en un análisis específico sobre los sectores (Gráfico 2), observamos que tanto los actores académicos con los estatales se encuentran fuertemente vinculados entre sí (aunque en menor medida los últimos). Cabe mencionar que la discriminación de nodos en los sectores académico y estatal responde a una clasificación basada en la función principal de cada persona u organismo en relación con el desarrollo de la innovación, ya que, en términos relativos a la esfera económica, ambos sectores corresponden al sector público. En contraposición al grado de cohesión de las redes académica y estatal, la red de actores privados se presenta con pocos nodos, con relaciones lejanas y aislados unos de otros.
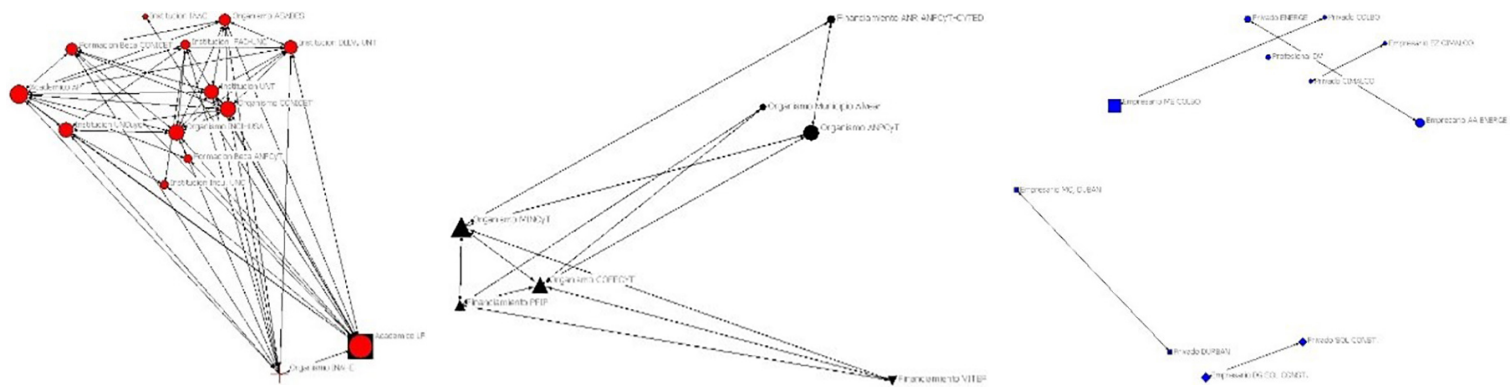

Gráfico 2: Análisis por sectores. Izquierda: Académico. Centro: Estado. Derecha: Privados.

Con respecto a las contribuciones realizadas por los nodos en torno a la concreción de la innovación, encontramos que para el atributo Aporta know-how se conforman dos redes en función de su análisis como un aporte directo o indirecto (Gráfico 3).

La red de aporte directo de know-how está integrada por apenas cuatro nodos, tres de ellos pertenecientes al sector privado y uno perteneciente al sector académico. Estos nodos tienen aportes "compuestos", complementando el know-how con tareas de fabricación, gestión, y financiamiento. Por otro lado, la red de aporte indirecto de know-how se presenta mucho más compleja, dando cuenta de la cantidad de actores y recursos del sector académico implicados en la generación de conocimientos necesarios para soportar el desarrollo 


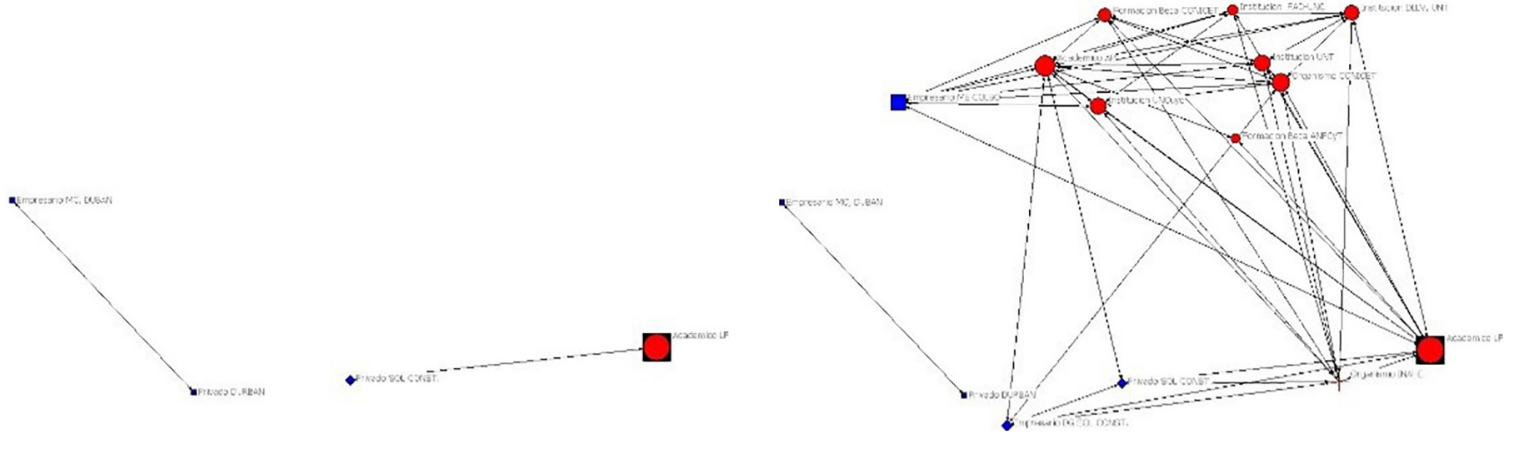

Gráfico 3: Análisis por atributo. Izquierda: Know-how directo. Derecha: Know-how indirecto.

del producto innovador. En ambas redes aparece como elemento común el nodo Académico $L F$, cuyo aporte conjuga know-how y gestión, confiriéndole el mayor grado de centralidad de la red. Adicionalmente, si bien excede los objetivos de este trabajo, sería interesante hacer un análisis cualitativo de los conocimientos directos aportados por cada una de las partes, a fin de sopesar su incidencia el proceso de innovación.

Finalmente, analizamos los nodos Programas de Formación y Organismos de Finamiento en relación con los nodos pertenecientes al sector Privado y el sector Académico (Gráfico 4). En un análisis comparativo encontramos que la red de instrumentos de financiamiento posee un mayor grado de centralidad cuando se trata del sector académico que con el sector privado. Esto puede representar un contrasentido desde la perspectiva del desarrollo de la innovación, ya que como vimos mediante el análisis de la red general (Gráfico 1), el subgrupo con mayor presencia de nodos académicos es el que menos aportes realiza.
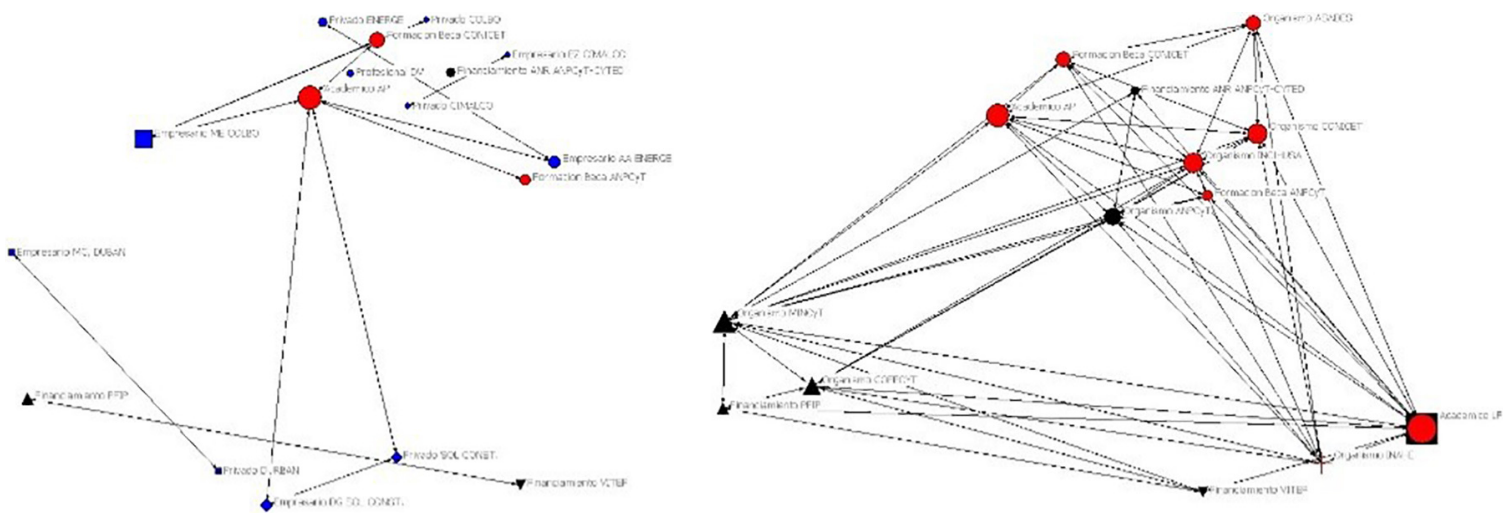

Gráfico 4: Izquierda: red privados - financiamiento - programas de formación. Derecha: red academia - financiamiento - programas de formación.

En relación con las métricas de análisis para cada uno de los actores se observa que los niveles de centralidad más elevados dentro de la red corresponden a nodos pertenecientes al sector académico, encabezados por los nodos LF (25), AP (17) e INAHE (16), seguidos por nodos pertenecientes al sector estatal, MINCyT (16). En estos nodos altamente conectados podemos encontrar atributos definidos por el aporte de Know-how+Gestión, Know-how indirecto y financiamiento indirecto.
En contraposición, los valores de centralidad más bajos recaen sobre nodos del sector privado: DURBAN (1), CIMALCO (1) y COLBO (1), actores entre cuyos atributos se cuenta el aporte de Know-How indirecto y la capacidad de fabricación. Finalmente, con un grado de centralidad intermedio encontramos actores del sector privado (SOL CONST. con 5) y programas estatales como el VITEF (4) y el PFIP (5), todos ellos con la característica de aportar financiamiento. 


\section{CONCLUSIONES}

El trabajo realizado da cuenta de la organización en clave relacional un conjunto de actores involucrados en el desarrollo de un producto innovador. A través de la metodología del ARS fue posible analizar la conformación de subgrupos de nodos cuyos atributos están asociados a las acciones de transferencia, desarrollo y financiamiento del producto. Asimismo, tanto las medidas de centralidad de los nodos como las medidas de coherencia de la red permitieron ordenar la información con el fin de evidenciar las vías de circulación de conocimiento. El análisis discriminado por atributos muestra que el sector académico se presenta como un subgrupo de nodos con relaciones estrechas y consolidadas, en tanto que los nodos directamente involucrados con el desarrollo y fabricación de la innovación forman un clúster con baja centralidad, cuyos actores están vinculados entre sí solo en torno al producto. Se observa además que los nodos cuyo aporte al desarrollo es el financiamiento - directo y/o indirecto- tienen mayor grado de relación con el sector académico y estatal que con el sector privado. Este hallazgo permite hacer diferentes lecturas. Por una parte, habiendo identificado que el subgrupo responsable de la producción del desarrollo está conformado principalmente por empresas y empresarios, el flujo de financiamiento hacia el sector académico puede representar un contrasentido como política en la promoción de innovaciones. No obstante, por otra parte, encontramos que el actor con mayor grado de centralidad de la red pertenece al sector académico y opera como puente que vincula los sectores académico y estatal - fuertemente relacionados hacia adentro y entre sí- con el sector privado - cuyos integrantes se encuentran aislados unos de otros-. Cabe destacar que este nodo se destaca dentro del subgrupo de actores académicos por conjugar como atributos el aporte de Know-How y de Gestión. Esto podría ser indicio de que la promoción de este tipo de perfiles dentro del sector académico, así como el diseño de herramientas de financiamiento orientadas a fomentar lazos entre actores con estas características y el sector productivo podría traccionar el desarrollo de productos innovadores con base en conocimiento científico.
Finalmente, este trabajo intenta ser un aporte más al conjunto de publicaciones que utiliza el análisis de redes sociales como metodología y herramienta para la conformación de redes y el estudio los flujos de información cuali-cuantitativa entre actores pertenecientes a diferentes ámbitos.

\section{Conflicto de intereses}

No hay conflicto de intereses en torno al manuscrito presentado.

\section{Declaración de consentimiento de datos}

Los datos generados durante el desarrollo de este estudio han sido debidamente incluidos en el manuscrito.॰

\section{REFERENCIAS}

Arocena, R., \& Sutz, J. (2005). Latin American universities: From an original revolution to an uncertain transition. Higher Education, 50, 573-592.

Brimble, P., \& Florida, R. (2007). University-Industry Linkages and Economic Development: The Case of Thailand. World Development, 35(6), 1021-1036.

Cohen, M., Nelson, R., \& Walsh, J. (2002). Links and Impacts: The Influence of Public Research on Industrial R\&D2. Management Science, 48(1), 1-23.

de la Torre, E. M., Agasisti, T., \& Perez-EsPARRELls, C. (2017). The relevance of knowledge transfer for universities' efficiency scores: an empirical approximation on the Spanish public higher education system. Research Evaluation, 26(3), 211-229.

Etzkowitz, H., \& LeydesdorfF, L., (2000). The dynamics of innovation: from National Systems and "Mode 2" to a Triple Helix of university-industry-government relations. Research Policy, 29, 109-123.

Galindo, J., Sanz, P., \& De Benito, J. (2011). La Universidad ante el reto de la transferencia del conocimiento 2.0: Análisis de las herramientas digitales a disposición del gesto de transferencia. Investigaciones Europeas de Dirección y Economía de la Empresa, 111-126. 
Gulbranddsen, M., \& Slipersaeter, S. (2007). The Third mission of the entrepreneurial university model. In: Bonaccorsi. A. and Daraio, C. (Eds), Universities and Strategic Knowledge Creation. Specialization and Performance in Europe.

Laursen, K., \& Salter, A. (2004). Searching high and low: what types of firms use universities as a source of innovation? Research Policy, 33, 1201-1215.

Mansfield (1991). Academic research and industrial innovation. Research Policy, 20, 1-12.

Molas-Gallart, J. (2005). Definir, quantificar i finançar la tercera missió: un debat sobre el futur de la universitat. Coneixement $i$ societat, (7), 6-26.

Rosenberg, N. (1976). Perspectives on Technology. Cambridge: Cambridge University Press.

Segarra-Blasco, A., \& Arauzo-Carod, J. P. (2008). Sources of innovation and industry-university interaction: Evidence from Spanish firms. Research Policy, 37, 1283-1295.

Tether, B., \& TAjar, A. (2008). Beyond industry-university links: Sourcing knowledge for innovation from consultants, private research organizations and the public science-base. Research Policy, 37, 1079-1095. 